Biomaterialia

Elsevier Editorial System(tm) for Acta Manuscript Draft

Manuscript Number: AB-17-1175R2

Title: Controlling human corneal stromal stem cell contraction to mediate rapid cell and matrix organization of real architecture for 3-dimensional tissue equivalents

Article Type: Full length article

Keywords: Corneal tissue engineering; extracellular matrix; alignment; stem cells; cell contraction; collagen; RAFT

Corresponding Author: Dr. Alvena Kureshi, Ph.D

Corresponding Author's Institution: University College London

First Author: Dev Mukhey

Order of Authors: Dev Mukhey; James B Phillips; Julie T Daniels; Alvena Kureshi, Ph.D

Abstract: The architecture of the human corneal stroma consists of a highly organized extracellular matrix (ECM) interspersed with keratocytes. Their progenitor cells; corneal stromal stem cells (CSSC) are located at the periphery, in the limbal stroma. A highly organized corneal ECM is critical for effective transmission of light but this structure may be compromised during injury or disease, resulting in loss of vision. Re-creating normal organization in engineered tissue equivalents for transplantation often involves lengthy culture times that are inappropriate for clinical use or utilisation of synthetic substrates that bring complications such as corneal melting. CSSC have great therapeutic potential owing to their ability to 
reorganize a disorganized matrix, restoring transparency in scarred corneas. We examined CSSC contractile behavior to assess whether this property could be exploited to rapidly generate cell and ECM organisation in RAFT tissue equivalents (TE) for transplantation. Free-floating collagen gels were characterized to assess contractile behavior of CSSC and establish optimum cell density and culture times. To mediate cell and collagen organisation, tethered collagen gels seeded with CSSC were cultured and subsequently stabilized with the RAFT process. We demonstrated rapid creation of biomimetic RAFT TE with tunable structural properties. These displayed three distinct regions of varying degrees of cellular and collagen organization. Interestingly, increased organisation coincided with a dramatic loss of PAX6 expression in CSSC, indicating rapid differentiation into keratocytes. The organized RAFT TE system could be a useful bioengineering tool to rapidly create an organized ECM while simultaneously controlling cell phenotype. 


\section{*Manuscript File}

Controlling human corneal stromal stem cell contraction to mediate rapid cell and matrix organization of real architecture for 3-dimensional tissue equivalents

Dev Mukhey ${ }^{1}$, James Phillips ${ }^{2}$, Julie T Daniels ${ }^{1}$, Alvena K Kureshi ${ }^{1 *}$

${ }^{1}$ Ocular Biology \& Therapeutics, Institute of Ophthalmology, University

College London, London, United Kingdom. ${ }^{2}$ Biomaterials \& Tissue

Engineering, Eastman Dental Institute, University College London,

London, UK.

*current affiliation:

Department of Materials \& Tissue,

UCL Institute of Orthopaedics \&

Musculoskeletal Science, Royal National Orthopaedic Hospital, Stanmore, London, UK.

Corresponding author:

Dr Alvena Kureshi

Department of Materials \& Tissue

UCL Institute of Orthopaedics \& Musculoskeletal Science

Royal National Orthopaedic Hospital

Stanmore, London, UK

a.kureshi@ucl.ac.uk

Keywords: Corneal tissue engineering, extracellular matrix, alignment, stem cells, cell contraction, collagen, RAFT 
Acknowledgements:

Supported by grants from The Special Trustees of Moorfields Eye Hospital (London, United Kingdom), National Institute for Health Research (NIHR) Biomedical Research Centre at Moorfields Eye Hospital NHS Foundation Trust, and UCL Institute of Ophthalmology (London, United Kingdom).

\section{ABSTRACT:}

The architecture of the human corneal stroma consists of a highly organized extracellular matrix (ECM) interspersed with keratocytes. Their progenitor cells; corneal stromal stem cells (CSSC) are located at the periphery, in the limbal stroma. A highly organized corneal ECM is critical for effective transmission of light but this structure may be compromised during injury or disease, resulting in loss of vision. Recreating normal organization in engineered tissue equivalents for transplantation often involves lengthy culture times that are inappropriate for clinical use or utilisation of synthetic substrates that bring complications such as corneal melting. CSSC have great therapeutic potential owing to their ability to reorganize a disorganized matrix, restoring transparency in scarred corneas. We examined CSSC contractile behavior to assess whether this property could be exploited to rapidly generate cell and ECM organization in Real Architecture For 3D Tissues (RAFT) tissue equivalents (TE) for transplantation. Free-floating collagen gels were characterized to assess contractile behavior of CSSC and establish optimum cell density and culture times. To mediate cell and collagen organization, tethered collagen gels seeded with CSSC were cultured and subsequently stabilized with the RAFT process. We demonstrated rapid creation of biomimetic RAFT TE with tunable structural properties. These displayed three distinct regions of varying degrees of cellular and collagen organization. Interestingly, increased organization coincided with a dramatic loss of PAX6 expression in CSSC, indicating rapid differentiation into keratocytes. The organized RAFT TE system could be a useful bioengineering tool to rapidly create an organized ECM while simultaneously controlling cell phenotype. 


\section{INTRODUCTION}

The corneal stroma is an organized, dense and avascular structure that occupies more than $90 \%$ of the corneal thickness. It consists of orthogonally oriented, highly aligned arrays of collagen (Type I and V) lamellae with collagen fibrils of uniform diameter and inter-fibril spacing [1]. It is thought that this weave-like arrangement of collagen lamellae facilitates the optical transparency and structural integrity of the cornea; both features that are key to its function. These are governed by the complex interplay between a highly organized extracellular matrix (ECM) or 'stromal' layer and the resident keratocyte cells that maintain a healthy cornea. This normal physiological balance can become compromised due to corneal injury or disease, resulting in scarring and loss of optical clarity. Loss of tissue organization is a key feature associated with corneal scarring and currently causes corneal blindness in 10 million people worldwide [2].

Engineering corneal tissue equivalents with structural properties that mimic the ECM of the native corneal stroma can provide useful in vitro models to investigate cell-ECM interactions. There is also scope to use these tissue equivalents clinically, for therapeutic translation. However, the hierarchical ultrastructure is complex and difficult to reproduce. A variety of techniques have been used to successfully align collagen, including the use of magnetic fields [3], electro-spinning of synthetic substrates [4] and the use of hydrodynamic flow [5]. However, these existing methods often involve high costs and lengthy preparation times. The implantation of synthetic substrates such as the KPro brings complications such as extrusion and corneal melting [6] so alternatives strategies are urgently required [7].

More recently, we have developed a rapid, GMP compliant, reproducible technique to engineer a corneal tissue equivalent using cell-seeded, collagen type I hydrogels stabilized by a process called Real Architecture For 3D Tissues (RAFT)[8]. These tissue equivalents (TE) have improved structural and mechanical properties to collagen hydrogels [9], resembling those observed in native tissue. However, RAFT TE have a random 
arrangement of collagen fibrils and any cells seeded within RAFT TE are distributed heterogeneously. A biomimetic substrate that mimics the highly organized arrangement of collagen fibrils of the corneal stroma interspersed with

keratocytes, would be the optimal tissue engineering approach. However, keratocytes are typically difficult to culture in vitro as they do not proliferate and if cultured in the presence of serum (as routinely done for corneal tissue engineering research), they quickly differentiate into fibroblasts; a wound-healing like phenotype undesired for bioengineering normal corneal tissue.

A population of corneal stromal stem cells (CSSC) has been identified in the human corneal limbal stroma as progenitor cells for keratocytes [10]. These cells have therapeutic potential as they have the capacity to reorganize a disorganized matrix and restore transparency in corneas from lumican-deficient mice [11]. They also proliferate rapidly in vitro, enabling clinically relevant large-scale culture without spontaneous differentiation into keratocytes or fibroblasts.

To create a model with increased structural similarity to the native cornea, the phenomenon of cellular self-alignment in tethered collagen gels was utilized [12][13]. Cells embedded within compliant collagen gels attach to the surrounding collagen fibrils via integrins causing cell movement and generation of force which contracts the gel. Subsequent stabilisation of tethered gels with the RAFT process increases the strength of gels whilst maintaining cell and collagen alignment, enabling their potential use for therapeutic translation [14]. This approach has been described in literature for the generation of aligned tissue equivalents for a variety of applications including peripheral nerve repair [15]. However, to our knowledge, this is the first study in which cellular self-alignment in a tethered collagen gel has been investigated in corneal cells.

In this study, we aimed to exploit the therapeutic potential of CSSC and investigated the feasibility of utilizing CSSC to develop an organized corneal stromal tissue equivalent. 


\section{METHODS}

Isolation and culture of CSSCs

Human CSSCs were isolated from three different organ-culture stored human donor corneal rims obtained from the Moorfields Lions Eye Bank (UK). All corneal tissue was obtained with research consent and ethical approval (ethics number: 10/H0106/57- 11ETR10). Human donor corneas were transferred from storage media and washed in Dulbecco's modified Eagle's medium (DMEM) and Ham's F12 medium (DMEM/F12) (Gibco) supplemented with $100 \mathrm{IU} / \mathrm{ml}$ penicillin (CellGro), $100 \mathrm{~g} / \mathrm{ml}$ streptomycin (CellGro). The superficial limbal stroma was dissected and digested overnight in DMEM/F12 containing $0.5 \mathrm{mg} / \mathrm{ml}$ collagenase type $\mathrm{L}$ (Sigma-Aldrich) at $37^{\circ} \mathrm{C}$ and $5 \% \mathrm{CO}_{2}$. The digested cell suspension was centrifuged and the cell pellet re-suspended in CSSC media. CSSC media consisted of DMEM/MCDB-201 (Sigma-Aldrich) with 2\% fetal bovine serum (FBS) (Invitrogen Corporation, Carlsbad, CA), $10 \mathrm{ng} / \mathrm{ml}$ epidermal growth factor (Sigma-Aldrich), $10 \mathrm{ng} / \mathrm{ml}$ platelet derived growth factor (PDGF-BB) (Sigma-Aldrich), $5 \mathrm{~g} / \mathrm{ml}$ insulin, $5 \mathrm{~g} / \mathrm{ml}$ transferrin, $5 \mathrm{ng} / \mathrm{ml}$ selenous acid (ITS) (Invitrogen), linoleic acid-bovine serum albumin (LA-BSA) (Gibco), $0.1 \mathrm{mM}$ ascorbic acid-2-phosphate, 10-8 M dexamethasone (Sigma-Aldrich), $100 \mathrm{IU} / \mathrm{ml}$ penicillin (CellGro), 100 $\mathrm{g} / \mathrm{ml}$ streptomycin (CellGro) and $50 \mathrm{~g} / \mathrm{ml}$ gentamicin (Gibco). Cells were cultured on flasks pre-coated with fibronectin and collagen (FNC) (AthenaES) and passaged using Tryple Express (Invitrogen, Paisley, UK) when cells reached $60 \%$ confluence. Media was changed three times a week.

Preparation of free-floating collagen gels

Gels were prepared on ice (to prevent premature fibrillogenesis) by mixing $80 \%$ rat- tail collagen type I (First Link, Birmingham, UK), 10\% of 10x Minimum Essential Medium (MEM) (Invitrogen, Paisley, UK) and 5.9\% Neutralizing Solution (TAP Biosystems, Royston, Hertfordshire, UK). The cells were added in a volume of $4.1 \%$ in DMEM at the required density. The solution was kept on ice for a further 30 minutes to allow 
dispersion of any air bubbles. Following this, $150 \mu \mathrm{l}$ of collagen solution was cast in a cylindrical well of a 48-well plate (Nunc). A minimum of three gels were prepared for each biological donor repeat. The plate was placed onto a heater (TAP Biosystems, Royston, Hertfordshire, UK) at $37^{\circ} \mathrm{C}$ for 15 minutes. Once the gels had set, $200 \mu$ of CSSC media was added to each well. Using a micropipette tip, the perimeter

and base of the gel was scored from the plastic well surround, leaving the gel completely free-floating. The gels were placed into the incubator at $37^{\circ} \mathrm{C}$ and $5 \% \mathrm{CO}_{2}$ and left to contract. At the end of the experiment, gels were fixed with $4 \%$ paraformaldehyde (PFA) for 30 minutes at room temperature, followed by 3 washes with PBS, prior to immunostaining.

Contraction Assay - free-floating collagen gels

To investigate whether CSSCs had the ability to align within a 3D extracellular matrix, it was important to first determine their capacity to contract a collagen matrix [16]. Firstly, a CSSC seeding density needed to be established. CSSCs were seeded into collagen hydrogels at various densities; $6.7 \times 10^{5}, 2 \times 10^{6}$, and $3.3 \times 10^{6} \mathrm{cell} / \mathrm{s} / \mathrm{ml}$ and cultured in CSSC media at $37^{\circ} \mathrm{C}$. These were allowed to contract and photographs of the contracted gels were taken at different time points. Magnified images were used to measure the area of the top surface of each gel using Image J software.

Immunohistochemistry

Cells were allowed to adhere on the chamber slides for approximately 4 hours prior to processing for immunohistochemistry. RAFT TE were washed with PBS prior to processing. Samples were blocked in 5\% goat serum, with the addition of $0.25 \%$ Triton X-100 (Sigma-Aldrich Ltd, Dorset, UK). This was followed with overnight incubation with primary antibody PAX6 at $4{ }^{\circ} \mathrm{C}$. Samples were washed in PBS and incubated for 1 hour at room temperature with secondary goat anti-rabbit 594 Alexa Fluor antibodies (1:500; Invitrogen Ltd, Paisley, UK). FITC-labeled phalloidin (Sigma- Aldrich Ltd, Dorset, UK) was added as a counterstain to 
highlight the actin cytoskeleton. Finally samples were mounted underneath coverslips in Vectashield Mounting Medium containing DAPI (Vector Laboratories Inc., Burlingame, CA) to counterstain nuclei before samples were viewed on a Zeiss LSM 710 microscope. Estimates of cell numbers expressing PAX6 were made by manually counting cells from 4 random locations on chamber slides (each aperture was approximately 100 cells) using Image $J$ analysis.

Preparation of organised RAFT TEs

A cell-seeded, tethered collagen gel was used to generate alignment of CSSCs and collagen fibrils. A polyether ether ketone (PEEK) mold (Figure 1) was used to cast collagen gels using the method described by Phillips and colleagues [15]. Briefly, $1.25 \mathrm{ml}$ of collagen solution was seeded with cells at a density of $6.7 \times 10^{5}$ cells $/ \mathrm{ml}$. This was pipetted into the mold ensuring the tethering bars were also immersed in collagen solution. Gels were placed on a heater at $37^{\circ} \mathrm{C}$ (TAP Biosystems, Royston, UK) to allow collagen gels to set for 15 minutes. The gel was submerged in $8 \mathrm{ml}$ of CSSC media and cultured for 8,24 or 72 hours at $37^{\circ} \mathrm{C}$ and $5 \% \mathrm{CO}_{2}$ to allow for contraction and alignment. At the end of culture, the gel was excised from the mold using a scalpel and placed on a petri dish. A hydrophilic porous absorber (TAP Biosystems) was placed on to the surface of the gel to allow stabilisation of the gel. This formed the organised RAFT TE with dimensions similar to the original mold size at approximately $16 \mathrm{~mm} \times 6 \mathrm{~mm} \times 1 \mathrm{~mm}$.

Calculating cell alignment

Confocal microscopy was used to capture tile scans for organised RAFT TE. Three images per region that represented the delta, edge and middle regions of organised RAFT TE (figure 1b) were analysed using Volocity software (Improvision/Perkin Elmer). The software was able to recognise individual cells and calculate their alignment to the long axis by mapping the overall orientation of the cell cytoskeleton (stained with phalloidin).

Scanning Electron Microscopy (SEM) 
Samples were fixed with 4\% paraformaldehyde (PFA) immediately following RAFT process and subsequently washed and stored in PBS until required for analysis. Samples were dehydrated in a graded series of alcohol concentrations $(20 \%, 50 \%, 70 \%$, and $90 \%)$ followed by $100 \%$ alcohol. Samples were placed in hexadimethylsilane for 2 minutes and then transferred to the desiccator. Once dry, samples were mounted on to aluminum stubs and were sputter coated with gold- palladium using a Polaron E5000 sputter coater. The specimens were viewed with a Cambridge 90B scanning electron microscope $(15 \mathrm{kV})$.

Reflection Confocal Microscopy

Reflection confocal microscopy (Axiovert S100/Radiance 2000) was used to visualize co-alignment of CSSC and collagen fibrils in organized RAFT TE. This technique generates high contrast optical section images of cells and collagen fibers from within 3-D matrices without the need for exogenous labeling. Samples were mounted on a glass slide underneath a glass cover-slip. Images were taken of each region of RAFT TE with a standard objective (Plan Neo-Fluar 60x 0.5NA, Zeiss). Simultaneous reflection and transmitted light images were acquired in parallel as $\mathrm{z}-$ stacks in $3 \mu \mathrm{m}$ sections through the depth of the sample. The acquired $\mathrm{z}$ stacks were imported into Volocity software program and were compressed into a single projection image.

Statistical analysis

A minimum of 3 RAFT TEs were fabricated per biological donor replicate. For the 8 hour experiment, box and whisker plots show variation of cell alignment in all experiments from $n=3$ biological donor replicates. Mean values were calculated for each region using data from three RAFT TEs per donor. Statistical analyses were performed using GraphPad Prism. To determine statistical differences in cell alignment in the varying regions of organized RAFT TE, a one-way ANOVA with Dunnet's posttest using delta region as a control was performed. 
For 24 and 72 hour experiments, box and whisker plots show data from 3 RAFT TEs for $\mathrm{n}=1$ biological donor replicate.

\section{RESULTS}

\section{Contraction Assay}

Human CSSC demonstrated a capacity to contract free-floating collagen gels when seeded at varying densities (figure 2). These were used to determine cell density and culture time required for RAFT TE culture experiments. It was expected that a cell density that contracted freefloating gels to $60-80 \%$ of their original size was required in order to observe significant alignment of cells in a tethered gel [16]. Following 4 hours of culture, CSSCs demonstrated a capacity to contract gels by over $50 \%$, increasing to approximately $90 \%$ by 24 hours. No further increase in contraction was observed beyond 24 hours. The free-floating gels were noted to contract symmetrically, with the central area appearing more transparent than the perimeter (data not shown). Since an average gel contraction of $60 \%$ was achieved with $6.7 \times 10^{5}$ cells $/ \mathrm{ml}$ in 8 hours, these parameters were chosen for aligned RAFT TE experiments.

\section{Cell alignment}

Cells in fully hydrated tethered gels were cultured for 8, 24 and 72 hours to allow contraction prior to RAFT stabilization. The resulting organized RAFT TEs were analysed for cell alignment, with 3 images taken per region. Representative confocal images of the different regions of organized RAFT TE (figure 3) illustrate the field of view from which percentage of cell alignment was calculated (cell number varied in each region due to contraction of gel. For example, collagen compaction occurred in the edge region leading to increased cell density in this area). A significant alignment of CSSC in the long axis was observed exclusively in middle and edge regions of organized RAFT TE, with an absence of cell alignment in the delta region following 
intrinsic control as cells are not subjected to the same orientated tension environment as elsewhere in the gel and therefore remain unaligned. Closer analysis revealed that at the edge and middle regions, $38 \%$ and $22 \%$ of cells respectively became maximally aligned (between $0^{\circ}$ and $10^{\circ}$ of the long axis). The delta region only demonstrated $10 \%$ of cells in this category. In contrast at the $80^{\circ}$ to $90^{\circ}$ bin category, where cells align perpendicular to the long axis, only $1 \%$ and $2 \%$ of cells at the edge and middle respectively were found at this orientation. This compares to $12 \%$ in the delta region, highlighting the difference in the degree of alignment between different regions of organised RAFT TE.

The distribution of cell alignment following 8, 24 and 72 hours in culture is summarized as box and whisker plots in figure 4. Figure 4a illustrates cell alignment after 8 hours of culture and the mean angle of orientation at the edge and middle are observed to be $12^{\circ}$ and $24^{\circ}$ (respectively) in deviation from the long axis. These regions were both significantly more aligned than the delta region that had a mean angle of orientation of $48^{\circ}$ $(\mathrm{p}<0.001$, one way anova with Dunnet's post-test using

Comparing the interquartile range from delta to middle to edge it can be seen to become more homogenous with respect to angle deviation. This was further enhanced following 24 and 72 hours of culture $(n=1$ biological donor replicates). As predicted, alignment of CSSC was not observed in the delta region.

Collagen alignment

To qualitatively assess the extent of collagen fibril alignment, scanning electron microscopy (SEM) was carried out on standard RAFT TE and on aligned RAFT TE. Figure 5a illustrates that standard RAFT TE appears to have a random arrangement of collagen fibrils. This was similar to the delta region of aligned RAFT TE that served as an intrinsic control of disorganized ECM (Supplementary figure 3). At the edge regions of organized RAFT TE, SEM analysis revealed dramatic alignment of collagen fibrils parallel to the long axis (fig 5b). The overall structure appears to resemble that of individual collagen lamellae layers where 
fibrils are bundled tightly together in a parallel array.

Cell and collagen fibril co-alignment

Reflection confocal microscopy was used to further assess whether cellular alignment coincided with collagen fibril alignment within organized RAFT TE (figure 6). After 8 hours of culture followed by RAFT stabilization, collagen fibrils appeared to co-align with CSSC parallel to the long axis of organized RAFT TE in middle and edge regions exclusively with a lack of alignment visible in the delta regions.

\section{Assessment of CSSC phenotype}

CSSC phenotype was characterized with PAX6; an early eye development transcription factor. Immunostaining of standard RAFT TE revealed that majority of cells expressed PAX6 and did not display any significant cell alignment (Supplementary figure 1). Following 8 hours of culture and RAFT stabilization of tethered gels, CSSC exhibited a dramatic loss of PAX6 expression (from approximately $96 \%$ to only 5\%) in all regions of aligned RAFT TE (figure 7 and Supplementary figure 1) indicating a loss in CSSC phenotype and possible differentiation into keratocytes. Further assessment of cell phenotype in organized RAFT TE demonstrated positive expression of keratocyte marker ALDH1A1, confirming a shift in phenotype following cell and collagen alignment (Supplementary figure 2).

\section{DISCUSSION}

We investigated whether the phenomenon of cellular self-alignment was exhibited by corneal stromal stem cells with the view to exploit this property in vitro to improve the architecture of engineered RAFT tissue equivalents of the corneal stroma. We found that CSSC exhibited a capacity to self-align in tethered collagen gels. This property of cellular self-alignment has not been previously demonstrated for CSSC using this tethered collagen system and represents a novel and rapid fabrication method for the manufacture of organized tissue equivalents. It was also observed that cell alignment mediated rapid co-alignment of collagen 
fibrils in just 8 hours of culture. This cellular and collagen fibril alignment was retained following stabilization of gels with the RAFT process. Importantly, the resulting biomimetic RAFT tissue equivalent had structural as well as cellular alignment and was fabricated much more rapidly than existing techniques used to engineer corneal tissue equivalents [17] [18] [19] [20] making it an ideal method for clinical manufacture. Although these approaches have demonstrated some reproduction of stromal architecture [19], they are often extremely lengthy, taking up to 9 weeks of culture; a timeframe that would carry significant risk and cost for Good Manufacturing Practice (GMP). In comparison, organized RAFT TE takes less than 10 hours (8 hours tethering, plus time to set and stabilize the gel) making it a more attractive choice for clinical manufacture. Additionally, the RAFT system has been commercialized and is now a reproducible and robust technique to generate tissue equivalents [8].

Alignment of cells and matrix is particularly important in the cornea as it is crucial for governing the tissue's transparency and structural integrity. The contraction of cells within a collagen matrix aids cellular selfalignment - a phenomenon well established in different cell types including Schwann cells and dermal fibroblasts [21] [12] [13]. Thus, cellular self-alignment can be a useful bioengineering tool for the development of aligned tissue equivalents in a variety of applications. Cellular self-alignment occurs as cells seeded within collagen gels attach to collagen fibrils and begin to contract, exerting a stress to the surrounding collagen matrix. Cells align parallel to the long axis of the gel in response to this tension, resulting in co-alignment of

collagen fibrils. The tethering bars provide stress-shielding [12] creating triangular regions at either ends of the gel (delta regions). Here, cells are not subjected to the same orientated tension environment as elsewhere in the gel and therefore remain unaligned, serving as useful intrinsic control areas. Subsequent stabilization of collagen gels with the RAFT process created organized RAFT TE.

Tissue engineered scaffolds need to be fabricated with the correct spatial 
format since the molecular orientation of collagen plays an important role in regulating cell morphology and differentiation/cell signalling [22]. Previous studies have shown the importance of scaffold alignment in a variety of tissues including the cornea. Keratocytes have been shown to respond to topographical cues, particularly as in vivo they are located interspersed between aligned collagen lamellae in the corneal stroma. A study by Saeidi and colleagues [23] demonstrated that disorganised matrix interferes in the deposition of organized ECM in vitro by delaying the production of useful matrix or by preventing uniform remodelling. Therefore, for normal remodelling to occur post-transplantation, it is vital to have a tissue equivalent with an organized matrix that will provide the correct cues to instruct host cells to lay down normal, organized collagen.

The effect of spatial orientation of collagen plays an important role in regulating cellular responses in other tissues too. Tang and colleagues [22] used tendon sections carved at different angles to create distinct spatial arrangements of collagen fibrils to mimic the microenvironment found in vivo. Human mesenchymal stem cells (MSC) cultured directly on longitudinal sections adopted an elongated and aligned morphology and expressed a tenocyte-specific protein; tenomodulin, demonstrating that a specific orientation of collagen fibrils promoted differentiation of MSC into tenocytes.

Until now, differentiated corneal fibroblasts were typically used for corneal tissue engineering research. However, fibroblasts are more representative of a wound- healing like cell cultured in $10 \%$ serum. The advantage of the CSSC-RAFT system is that CSSC (precursors of keratocytes) are found in the native niche of healthy corneal stromal tissue and unlike keratocytes, rapidly proliferate in vitro and can be cultured to large quantities necessary for bioengineering research. CSSC also have unique re-

organising capabilities [11]. Previous studies similarly demonstrate that an aligned substrate may influence corneal cells to secrete a more organized ECM and a less light-scattering cellular phenotype [25]. Their study also demonstrated alignment as an important cell signal for down- 
regulating expression of alpha-smooth muscle actin (alpha-SMA). Decreased levels of alpha-SMA expression were linked to improved optical properties. Since alpha-SMA is a contractile protein it is thought to contribute to corneal haze in the wound-healing phase. Therefore, increased levels of expression are thought to increase light scattering. Since the CSSC-RAFT culture system utilizes only $2 \%$ serum, it is thought that fibrotic cell phenotype activation is unlikely to occur. Previous studies in our lab have demonstrated that corneal stromal fibroblasts cultured in RAFT TE with $10 \%$ serum do not express alpha smooth muscle actin [9], an indicator of a contractile, scarring phenotype that would be inappropriate for fabrication of normal tissue equivalents. We have also previously described the mechanical properties and optical clarity of RAFT TE [9] demonstrating their similarity to native cornea. We expect the material and optical properties of organized RAFT TE to be in a similar range to standard RAFT TE but as this assessment is beyond the scope of this manuscript, it will need to be carried out in future studies.

A novel finding in our study was that generation of cell and collagen fibril alignment appeared to accelerate CSSC differentiation within organised RAFT TE. A dramatic loss of PAX6 expression in CSSC following tethering occurred; a biomarker of CSSC differentiation into keratocytes. PAX6 is a well-known marker for CSSC and the loss of PAX6 is indicative of keratocyte phenotype [10] and we have previously validated CSSC and keratocyte markers [26]. Furthermore, immunofluorescence staining of ALDH1A1 in organized RAFT TE was observed, suggesting a switch to the keratocyte phenotype (Supplementary figure 2). A study by Jester and colleagues [27] suggests that identification of keratocyte phenotype can be accomplished through detection of cytoplasmic ALDH.

Further characterization of cell phenotype is needed but this suggests that alignment of RAFT TE may be a useful tool for rapidly differentiating CSSC to keratocytes (a quiescent, non-fibrotic phenotype, desirable for bio-engineering of normal tissue). A recent study by Li and colleagues [28] emphasizes the importance of two-way regulation between cells and 
aligned collagen fibrils in a different application of neural cell injury repair. The authors demonstrated that an aligned 3D matrix mediates neuronal differentiation of human decidua parietalis placental stem cells (hdpPSCs) through $\beta-1$ integrin. Future investigations will be required to elucidate the exact mechanisms regulating CSSC differentiation into keratocytes.

This study provides a novel and rapid method for fabrication of organized RAFT tissue equivalents of the corneal stroma. The resulting tissue equivalents have improved cell and collagen fibril alignment than standard RAFT TE and are fabricated in less than 10 hours making it a feasible approach for clinical manufacture. The microstructure is similar to a single native collagen lamellae and it is envisaged that these constructs could be used for partial thickness stromal repair or as a guide to instruct host cells to lay down organized matrix post-transplantation. Organized RAFT TE could also be used in vitro as a model to improve understanding of cell-ECM interactions governing tissue organization or further developed for use as a clinical therapy.

References

1. Meek K. M and Boote C. (2009) The use of X-ray scattering techniques to quantify the orientation and distribution of collagen in the corneal stroma. Prog Retin Eye Res. 28(5):369-92. doi: 10.1016/j.preteyeres.2009.06.005

2. Whitcher J. P., Srinivasan M., Upadhyay M. P. (2001) Corneal blindness: a global perspective. Bull. World Health Organ. 79 (3): 214-221

3. Wilson S. L., Guilbert M., Sulé-Suso J., Torbet J., Jeannesson P., Sockalingum G. D., Yang Y. (2014) A microscopic and macroscopic study of aging collagen on its molecular structure, mechanical properties, and cellular response. FASEB J. 28(1):1425. doi: 10.1096/fj.13-227579. 
4. Matthews J. A, Wnek G. E, Simpson D. G, Bowlin G. L. (2002) Electrospinning of collagen nanofibers. Biomacromolecules. (2):232-8.

5. Jiang F., Hörber H., Howard J., Müller D. J. (2004) Assembly of collagen into microribbons: effects of $\mathrm{pH}$ and electrolytes. J Struct Biol. 148(3):268-78.

6. Robert M. C, Dohlman C. H. (2014) A review of corneal melting after Boston Keratoprosthesis. Semin Ophthalmol. 29(5-6):349-57. doi: 10.3109/08820538.2014.959186.

7. Princz M. A., Sheardown H., Griffith M. (2009) Corneal tissue engineering versus synthetic artificial corneas, in Biomaterials and Regenerative Medicine in Ophthalmology, T. V. Chirila, Ed. (CRC Press/Woodhead Publishing, Cambridge, UK).

8. Levis H. J., Kureshi A. K., Massie I., Morgan L., Vernon A. J., Daniels J. T. (2015) Tissue Engineering the Cornea: The Evolution of RAFT. J Funct Biomater. 6(1):50-65. doi: 10.3390/jfb6010050.

9. Massie I., Kureshi A. K., Schrader S., Shortt A. J., Daniels J.T. (2015) Optimization of optical and mechanical properties of real architecture for 3-dimensional tissue equivalents: Towards treatment of limbal epithelial stem cell deficiency. Acta Biomater 24:241-50. doi: 10.1016/j.actbio.2015.06.007.

10. Du Y., Funderburgh M. L., Mann M. M, SundarRaj N., Funderburgh J. L. (2005) Multipotent stem cells in human corneal stroma. Stem Cells 23(9):1266-75.

11. Du Y., Carlson E. C., Funderburgh M. L., Birk D. E., Pearlman E., Guo N., Kao W. W., Funderburgh J. L. (2009) Stem cell therapy 
restores transparency to defective murine corneas. Stem Cells 27(7):1635-42. doi: 10.1002/stem.91.

12. Eastwood M., McGrouther D. A., Brown R. A. (1998) Fibroblast responses to mechanical forces. Proc Inst Mech Eng H. 212(2):85-92.

13. Tomasek J. J, Gabbiani G., Hinz B., Chaponnier C., Brown R. A. (2002) Myofibroblasts and mechano-regulation of connective tissue remodelling. Nat Rev Mol Cell Biol. 3(5):349-63.

14. Georgiou M., Bunting S. C., Davies H. A., Loughlin A. J., Golding J. P., Phillips J. B. (2013) Engineered neural tissue for peripheral nerve repair. Biomaterials. 34(30):7335-43. doi: 10.1016/j.biomaterials.2013.06.025.

15. Phillips J. B. (2014) Building stable anisotropic tissues using cellular collagen gels. Organogenesis. 1;10(1):6-8. doi: 10.4161/org.27487.

16. O'Rourke C., Drake R. A., Cameron G. W., Loughlin A. J., Phillips J. B. (2015) Optimising contraction and alignment of cellular collagen hydrogels to achieve reliable and consistent engineered anisotropic tissue. J Biomater Appl. 30(5):599-607. doi: $10.1177 / 0885328215597818$.

17. Guan L., Ge H., Tang X., Su S., Tian P., Xiao N., Zhang H., Zhang L., Liu P. (2013) Use of a silk fibroin-chitosan scaffold to construct a tissue-engineered corneal stroma. Cells Tissues Organs. 198(3):190-7. doi: 10.1159/000355944.

18. Fuest M., Yam G. H., Peh G. S., Mehta J. S. (2016) Advances in corneal cell therapy. Regen Med. 11(6):601-15. doi: 10.2217/rme2016-0054. 
19. Wu J., Du Y., Mann M. M., Yang E., Funderburgh J. L., Wagner W. R. (2013) Bioengineering organized, multilamellar human corneal stromal tissue by growth factor supplementation on highly aligned synthetic substrates. Tissue Eng Part A. 19(17-18):206375. doi: 10.1089/ten.TEA.2012.0545

20. Gouveia R. M., Hamley I. W., Connon C. J. (2015) Bio-fabrication and physiological self-release of tissue equivalents using smart peptide amphiphile templates. J Mater Sci Mater Med. 26(10):242. doi: 10.1007/s10856-015-5581- 5 .

21. Mudera V. C., Pleass R., Eastwood M., Tarnuzzer R., Schultz G., Khaw P., McGrouther D. A., Brown R. A. (2000) Molecular responses of human dermal fibroblasts to dual cues: contact guidance and mechanical load. Cell Motil Cytoskeleton. 45(1):1-9.

22. Tang S. W., Tong W. Y., Shen W., Yeung K. W., Lam Y. W. (2014) Stringent requirement for spatial arrangement of extracellular matrix in supporting cell morphogenesis and differentiation. BMC Cell Biol. 15:10. doi: 10.1186/1471- 2121$15-10$.

23. Saeidi N., Guo X., Hutcheon A. E., Sander E. A., Bale S. S., Melotti S. A., Zieske J. D., Trinkaus-Randall V., Ruberti J. W. (2012) Disorganized collagen scaffold interferes with fibroblast mediated deposition of organized extracellular matrix in vitro. Biotechnol Bioeng. 109(10):2683-98. doi: 10.1002/bit.24533.

24. Du Y., Carlson E. C., Funderburgh M. L., Birk D. E., Pearlman E., Guo N., Kao W. W., Funderburgh J. L. (2009) Stem cell therapy restores transparency to defective murine corneas. Stem Cells. 27(7):1635-42. doi: 10.1002/stem.91.

25. Phu D., Wray L. S., Warren R. V., Haskell R. C., Orwin E. J. (2011) Effect of substrate composition and alignment on corneal 
cell phenotype. Tissue Eng Part A. 17(5-6):799-807. doi: 10.1089/ten.TEA.2009.0724

28. Li W., Zhu B., Strakova Z., Wang R. (2014) Two-way regulation between cells and aligned collagen fibrils: local 3D matrix formation and accelerated neural differentiation of human decidua parietalis placental stem cells. Biochem Biophys Res Commun. 8;450(4):1377-82. doi: 10.1016/j.bbrc.2014.06.136.

26. Kureshi A. K., Funderburgh J. L., Daniels J. T. (2014) Human corneal stromal stem cells exhibit survival capacity following isolation from stored organ- culture corneas. Invest Ophthalmol Vis Sci. 55(11):7583-8. doi: 10.1167/iovs.14-14448

27. Jester J. V., Moller-Pedersen T., Huang J., Sax C. M., Kays W. T., Cavangh H. D., Petroll W. M., Piatigorsky J. (1999) The cellular basis of corneal transparency: evidence for 'corneal crystallins'. J Cell Sci. 112 ( Pt 5):613-22.

\section{FIGURE LEGENDS}

Figure 1: Fabrication of organized RAFT tissue equivalents (TE). (a) Photograph illustrating H-shaped plastic mould and tethering bars used to tether collagen gel, submerged in a petri dish containing cell culture media. (b) Diagram illustrating different regions of organized RAFT TE. The position of delta (D), middle (M) and edge (E) regions are shown. Arrow indicates direction of alignment/long axis.

Figure 2: Contraction profile of CSSC in free-floating gels. (a) Contraction profile of CSSC at different cell densities in free-floating collagen gels. Densities of $6.7 \times 10^{5}, 2 \times 10^{6}$, and $3.3 \times 10^{6} \mathrm{cells} / \mathrm{ml}$ were tested $(n=3)$. Gels contracted by $65 \%$ within 8 hours of culture at the lowest cell density. These parameters were chosen for aligned RAFT TE 
culture conditions. (b) Graph illustrating average percentage of gel contraction over initial 8 hours at cell density of $6.7 \times 10^{5}$ cells $/ \mathrm{ml}$.

Figure 3: Cell alignment in different regions of organized RAFT TE at 8 hours. Histogram illustrates percentage of cells at varying angles from the long axis. At the middle and edge regions, values are skewed towards $0^{\circ}$ - $10^{\circ}$ suggesting increased alignment, whilst the delta region has a relatively even distribution of cell alignment indicating lack of alignment. $(\mathrm{n}=3$ biological donor replicates, 3 RAFT TEs per donor and error bars indicate standard error mean).

Figure 4: Box and whisker plots comparing degree of cell alignment in all regions of organized RAFT TE at (a) 8 hours, (b) 24 hours, (c) 72 hours. Boxes highlight the mean angle of alignment, the upper and lower quartile range, with whiskers representing the limits of the 5\% and $95 \%$ extremities. At 24 hours and 72 hours, a lower angle of deviation from the long axis can be seen in the edge regions suggesting increased alignment in this region ( 3 RAFT TE analysed for $n=1$ biological donor replicate).

Figure 5: Scanning electron microscopy (SEM) images illustrating collagen fibril arrangement following 8 hours of culture in (a) standard RAFT TE and (B) organized RAFT TE. A disorganized arrangement of collagen fibrils is observed in standard RAFT TE, with no evidence of fibril alignment. Increased organization and collagen fibril bundling is observed in organized RAFT TE. Arrow shows direction of long axis of organized RAFT TE. Scale bar 2 um.

Figure 6: Paired reflection confocal microscopy images of cell and collagen fibril alignment in organized RAFT TE ( 8 hours). Delta region demonstrates random arrangement of cells (left panel) and collagen fibrils Co-alignment of cells and collagen fibrils can be seen in middle and edge regions.

Figure 7: Histogram illustrating percentage of CSSC expressing PAX6 in standard RAFT TE and different regions of organized RAFT TE. A dramatic loss of PAX6 expression was observed following 8 hours of 
culture in all regions of organized RAFT TEs, suggesting loss of CSSC phenotype.

Supplementary figure 1: Immunofluorescence expression of PAX6 in standard and organized RAFT TE. Confocal images illustrating assessment of PAX6 (CSSC marker) in standard RAFT TE and the different regions of organized RAFT TE following 8 hours of culture. (Blue - DAPI; Green- Phalloidin; Red - PAX6). Scale bar 20um. White arrow indicates long axis of organized RAFT TE. PAX6 expression is observed in standard RAFT TE but appears lost in organized RAFT TE.

Supplementary figure 2: Assessment of keratocyte phenotype of cells in organized RAFT TE. Confocal split channel images of delta, middle and edge regions of organised RAFT TE following 8 hours of culture. Cytoplasmic ALDH1A1 expression is observed in all regions of organized RAFT TE, confirming loss of CSSC phenotype and a switch to a keratocyte phenotype. (Blue - DAPI; Green- Phalloidin; Red ALDH1A1 - keratocyte marker). White arrow indicates long axis of organized RAFT TE.

Supplementary figure 3: SEM analysis of delta, middle and edge regions of organized RAFT TE at 8 hours. Delta region shows no evidence of alignment of collagen fibrils. Increased alignment is observed in middle and edge regions. Arrow shows direction of long axis of organized RAFT TE.

\section{*Statement of Significance}

Statement of significance

For the first time, we have demonstrated that human CSSC exhibit the phenomenon of cellular self-alignment in tethered collagen gels. We found this mediated rapid co-alignment of collagen fibrils and thus subsequently exploited this property in vitro to improve the architecture 
of engineered RAFT tissue equivalents of the corneal stroma. Existing techniques are extremely lengthy and carry significant risk and cost for GMP manufacture. This rapid and tunable technique takes just 8 hours of culture and is therefore ideal for clinical manufacture, creating biomimetic tissue equivalents with both cellular and ECM organization. Thus, cellular self-alignment can be a useful bioengineering tool for the development of aligned tissue equivalents in a variety of applications.

\section{${ }^{\star}$ Graphical abstract}

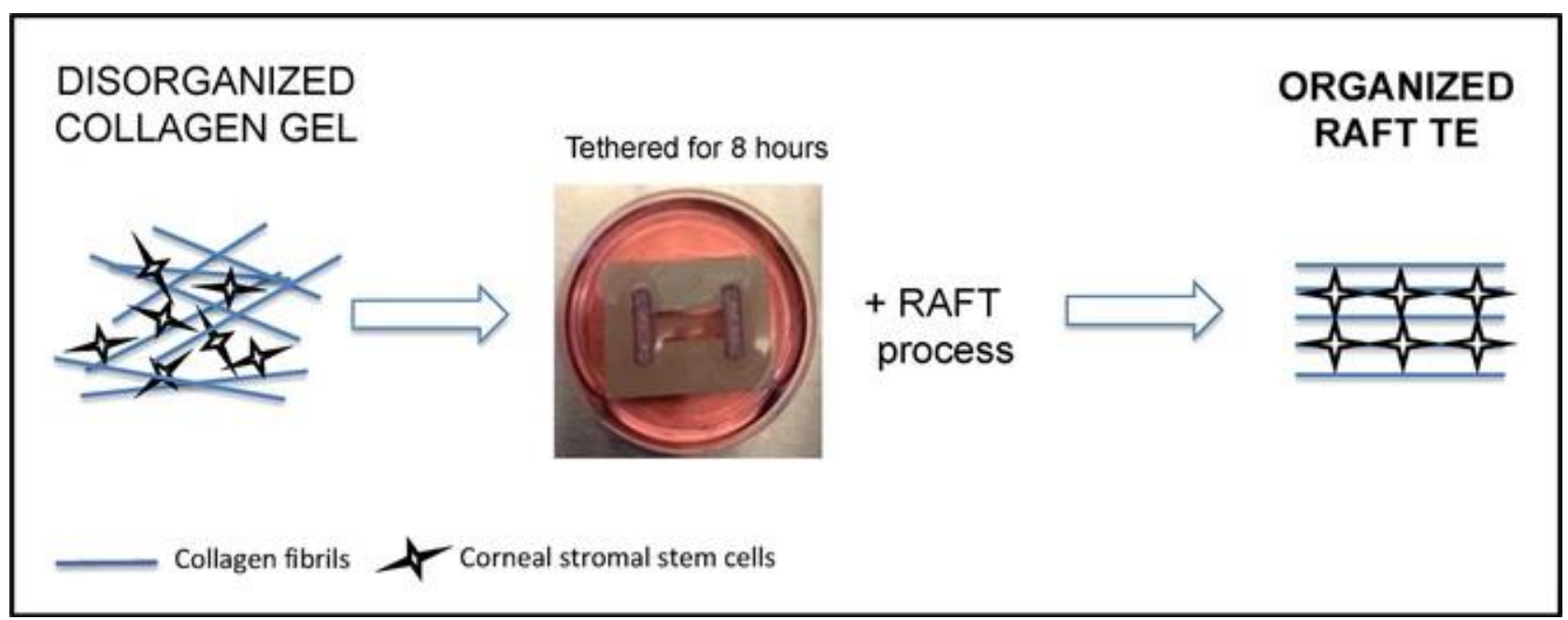


Figure 1
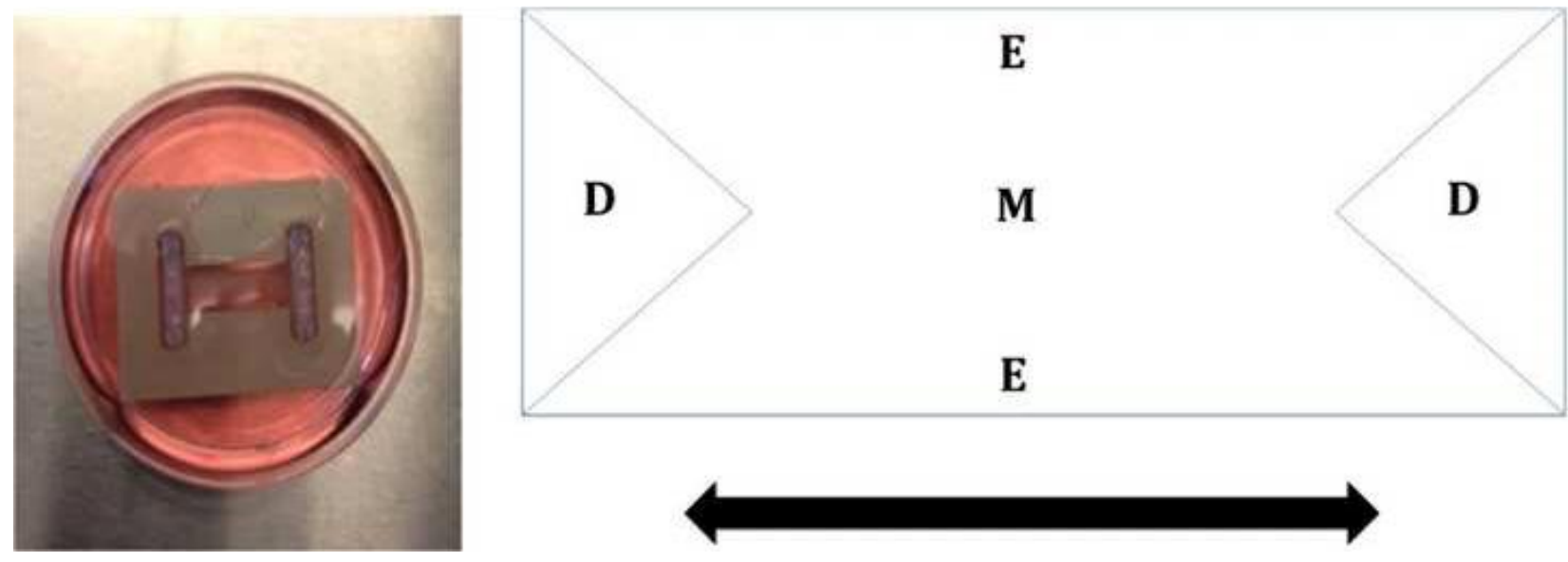

Figure 2 


\section{Contraction Assay}

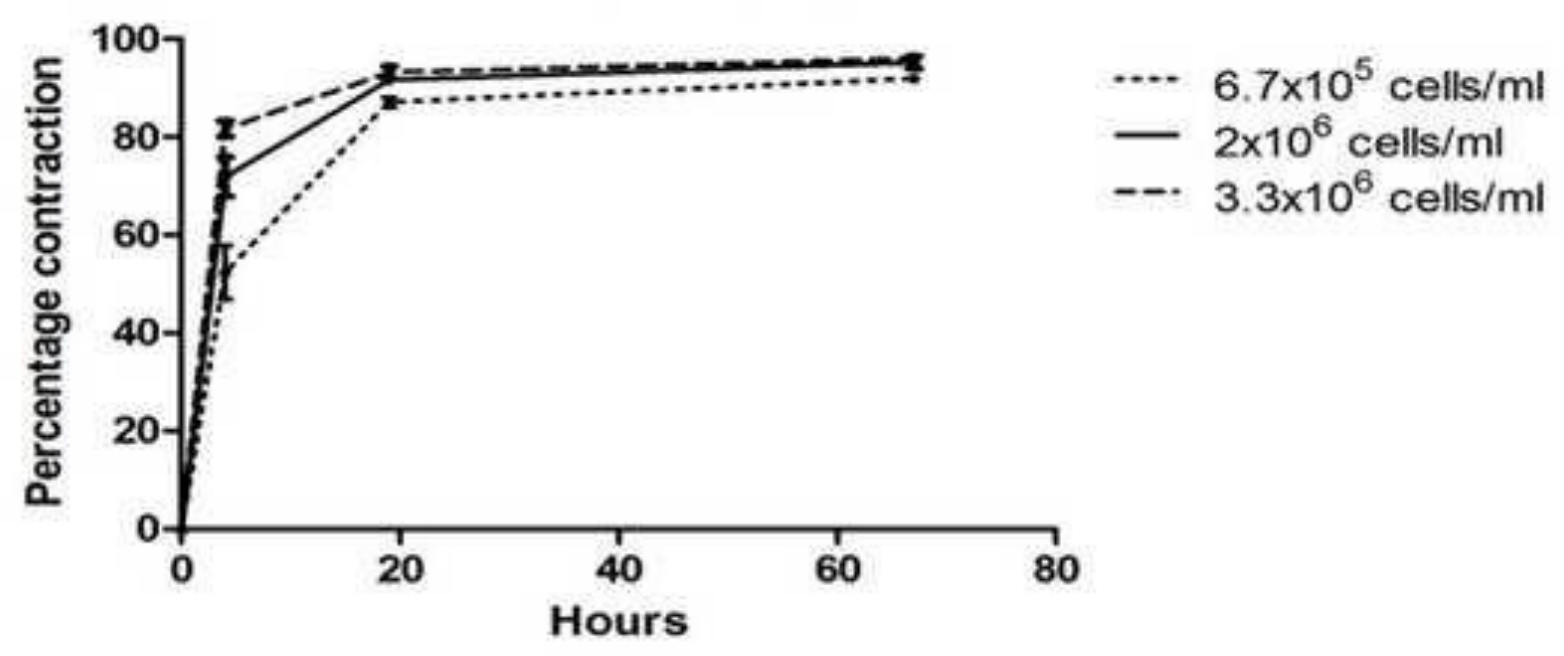

Mean percentage contraction of cells at $6.7 \times 10^{5} \mathrm{cells} / \mathrm{ml}$

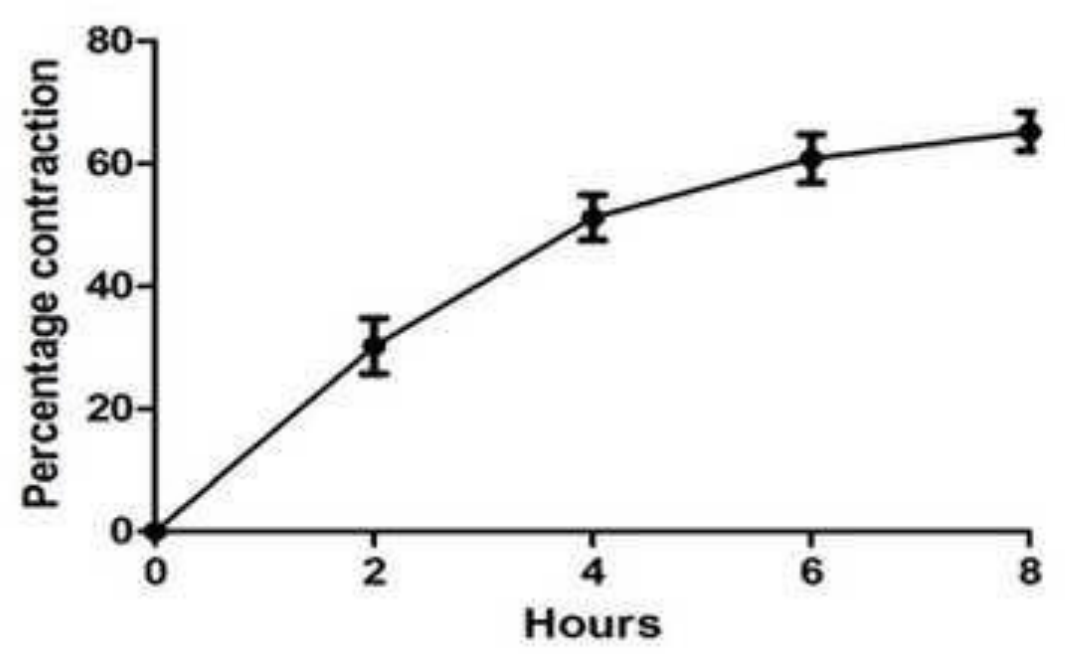


Figure 3
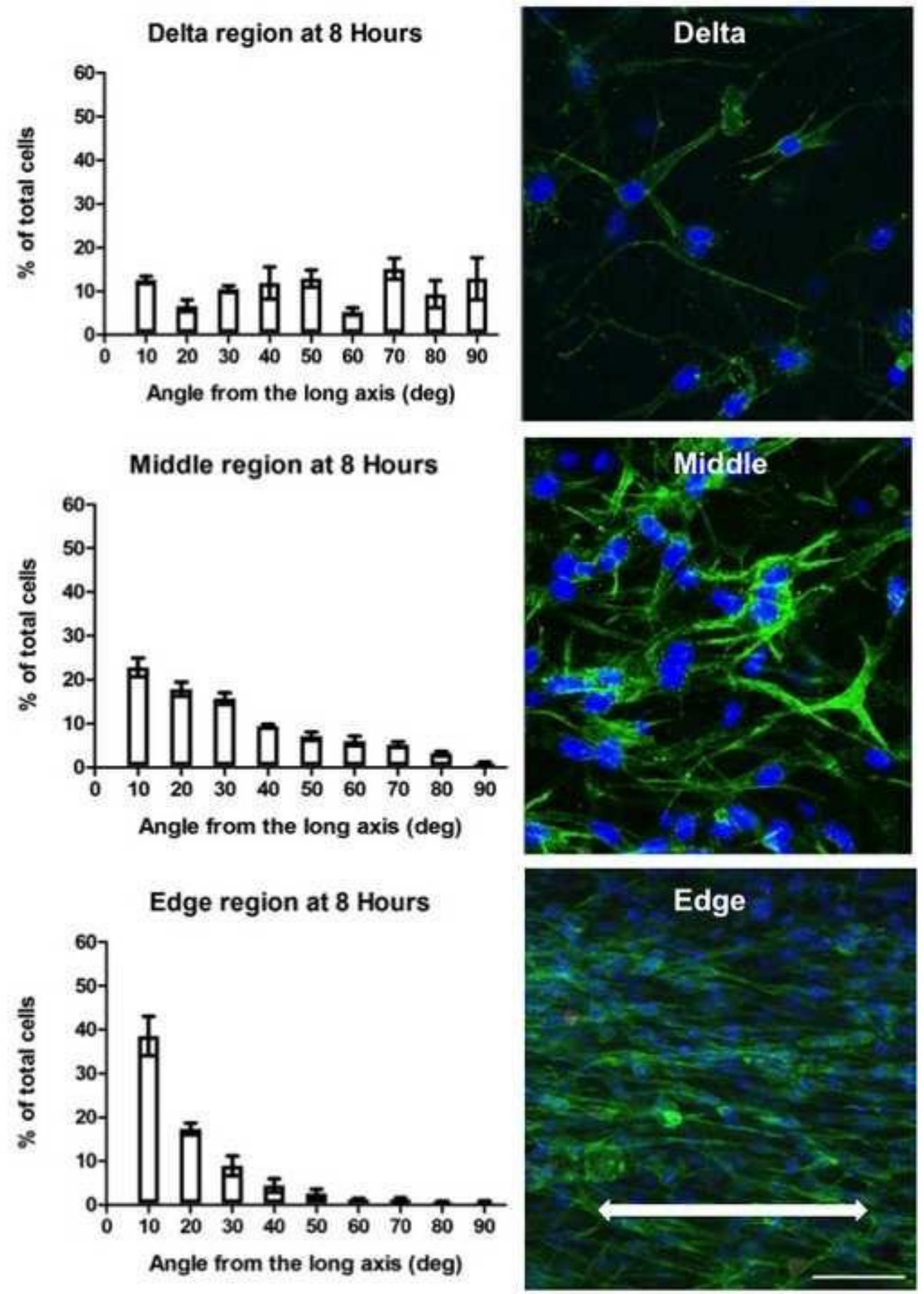

Figure 4 

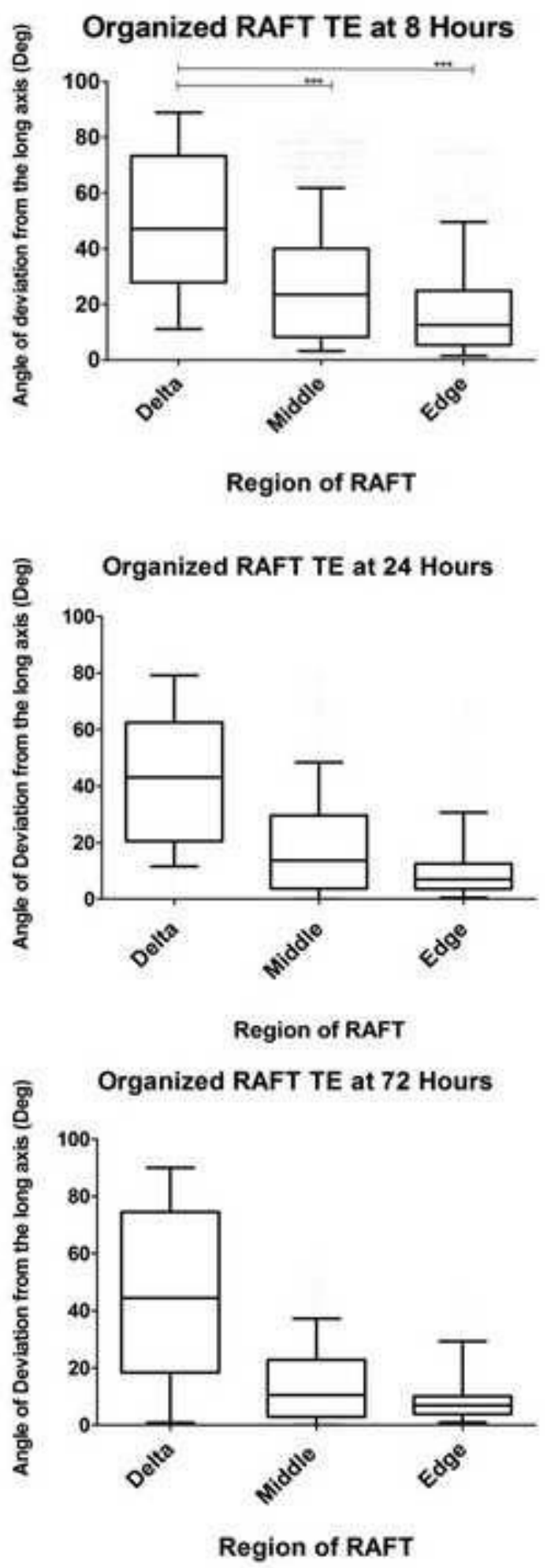

Figure 5 

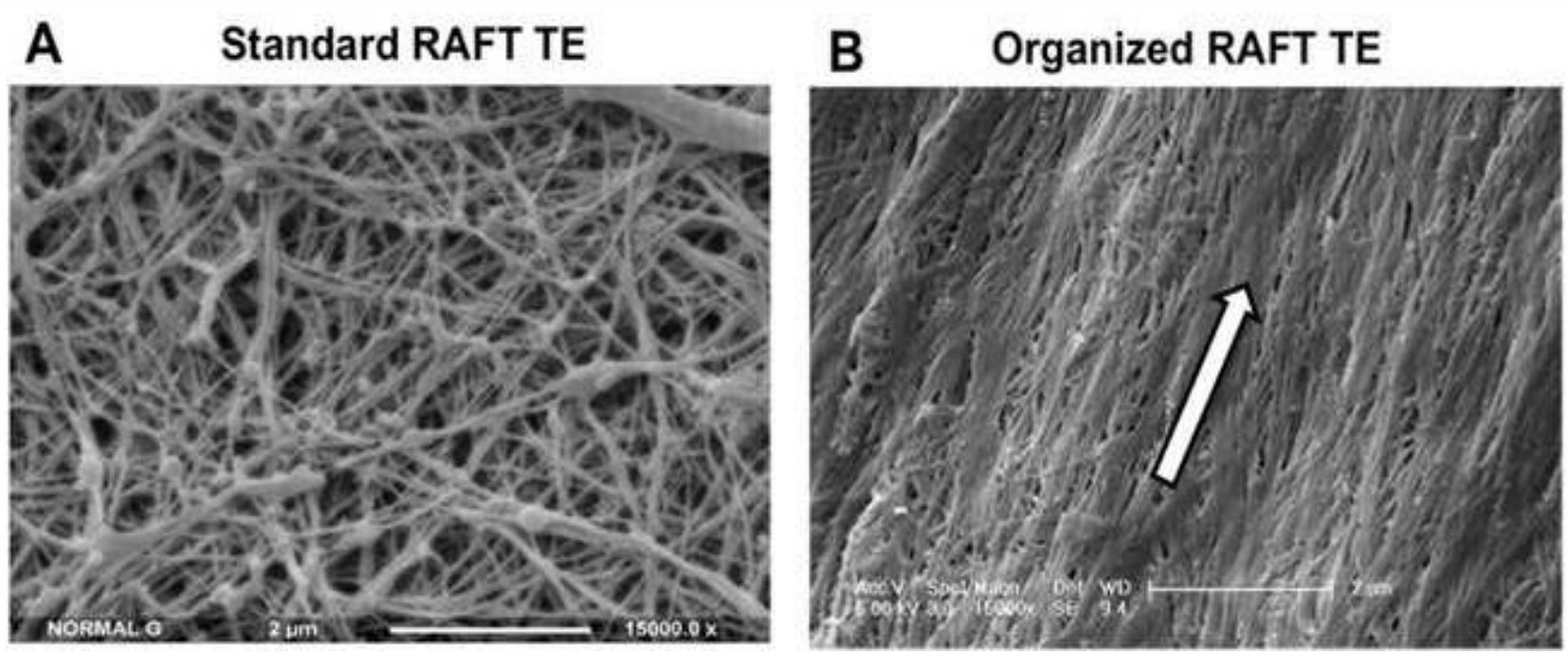

Figure 6 


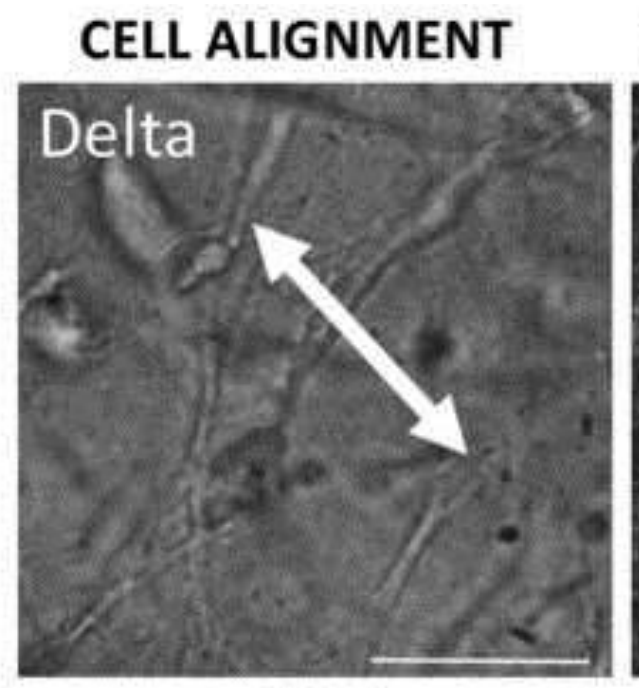

COLLAGEN ALIGNMENT
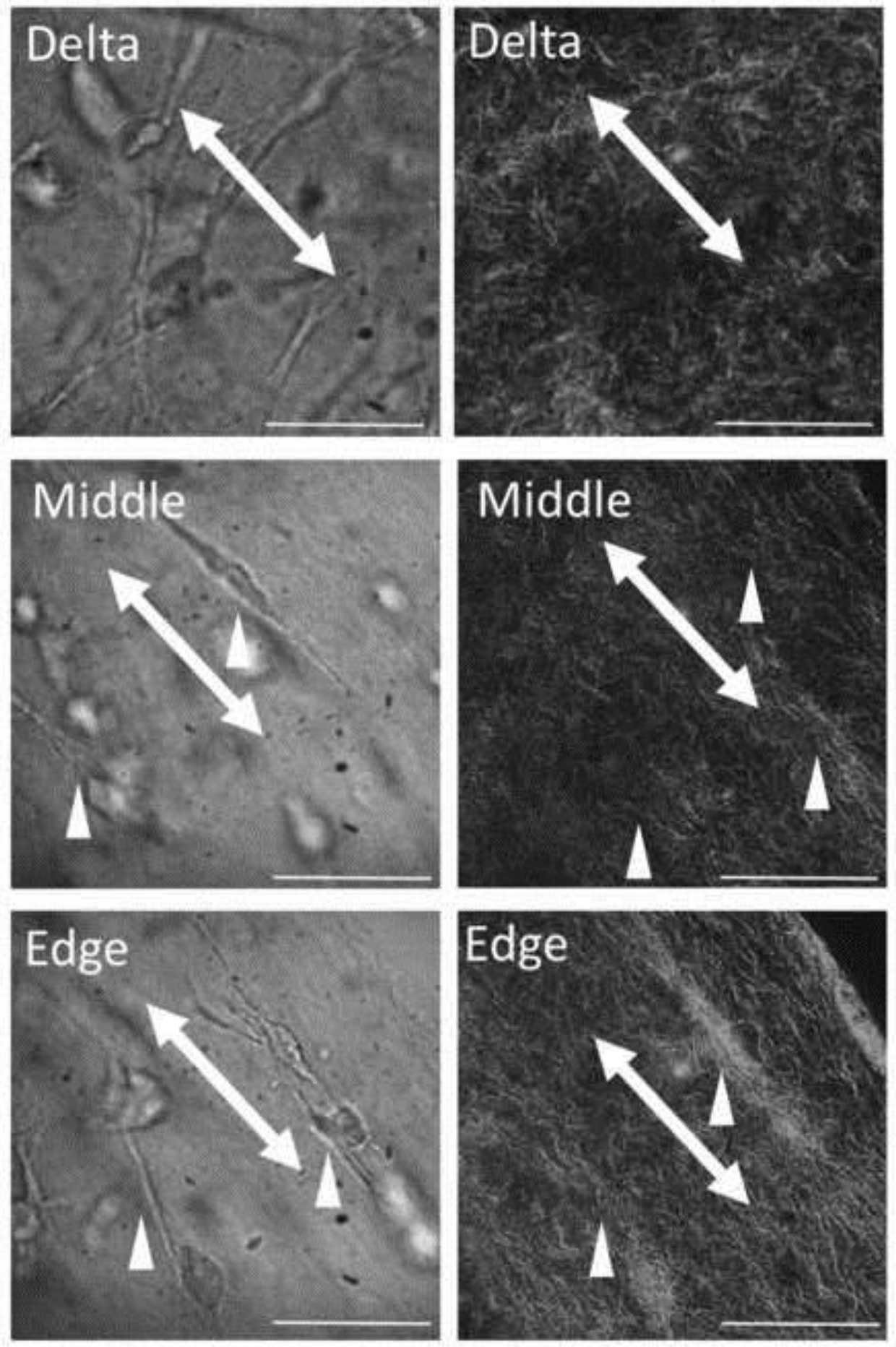

Figure 7 
PAX6 expression of CSSC at 8 hours

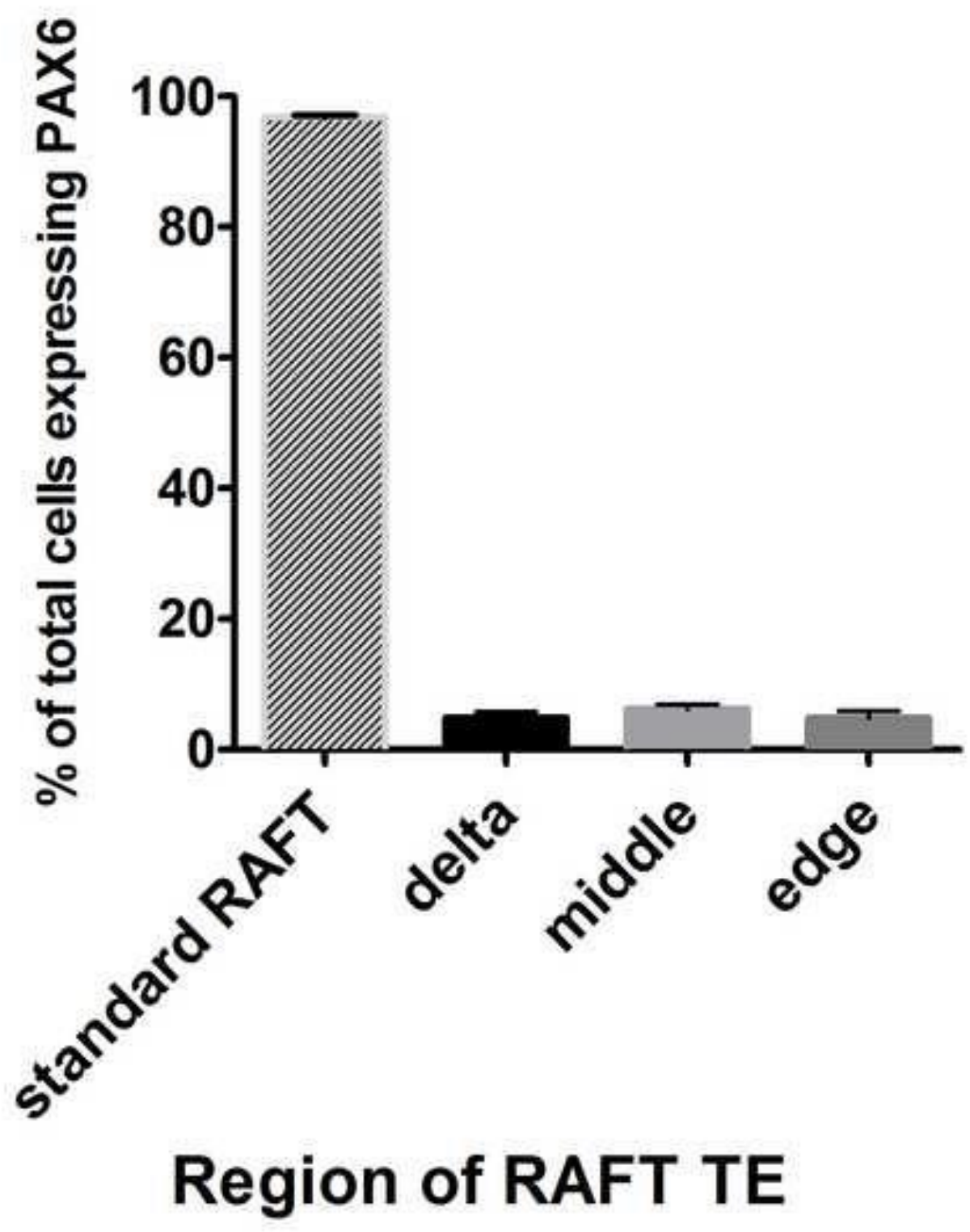


Supplementary Material - figure 1

Standard

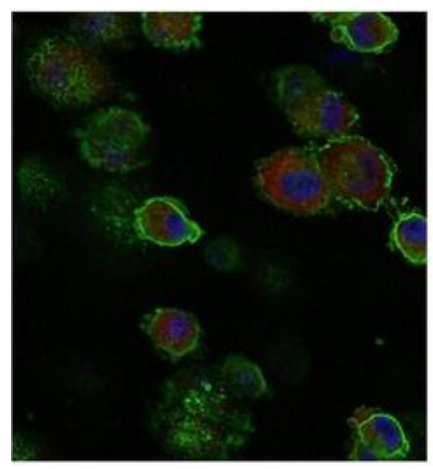

Delta

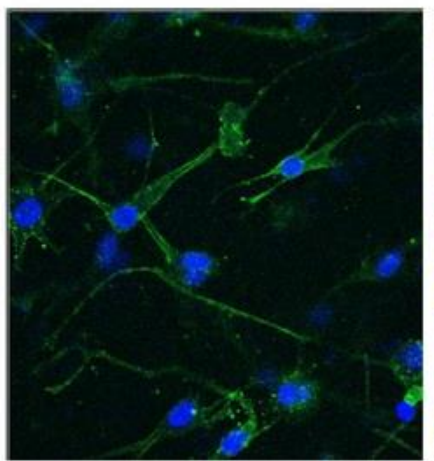

Middle

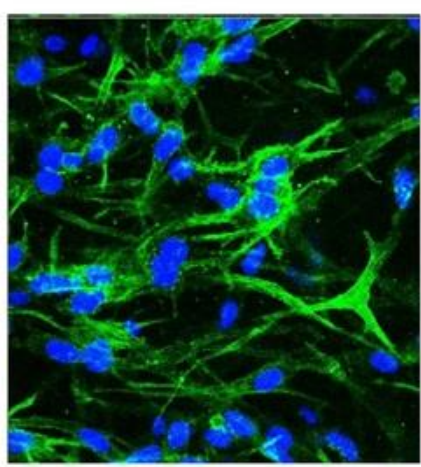

Edge

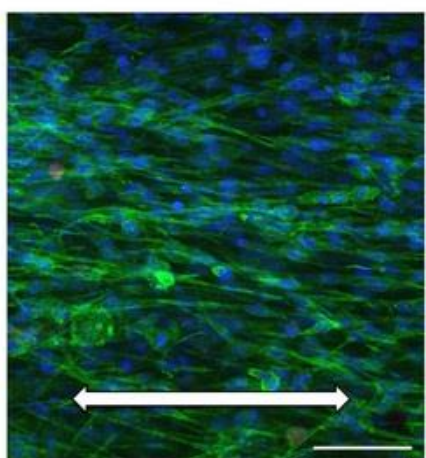

Supplementary Material - figure 2 
Delta

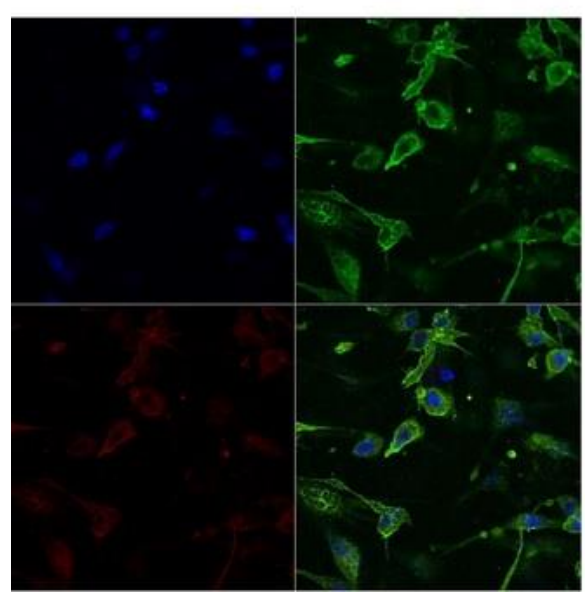

Middle

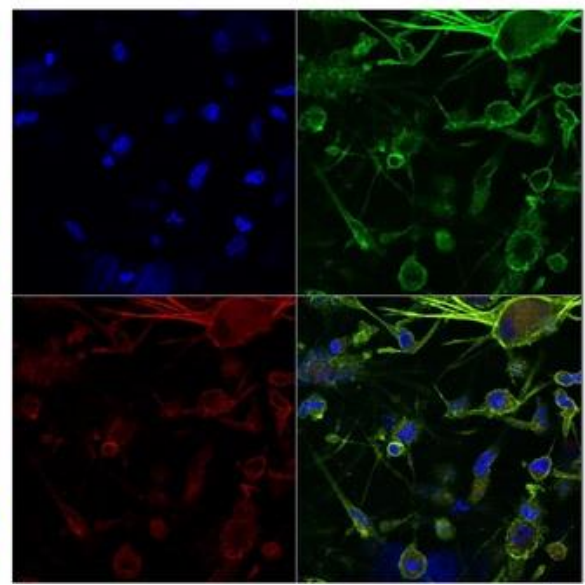

Edge

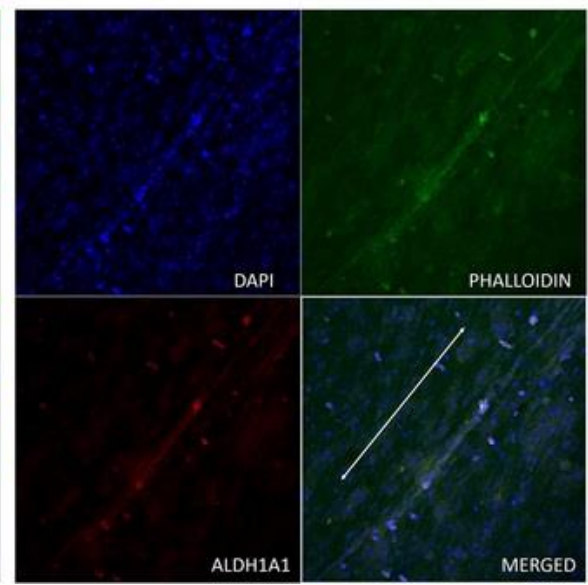

Supplementary Material - figure 3 
DELTA

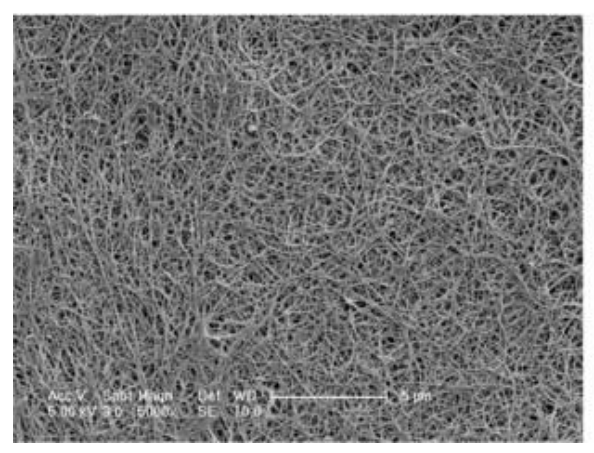

MIDDLE

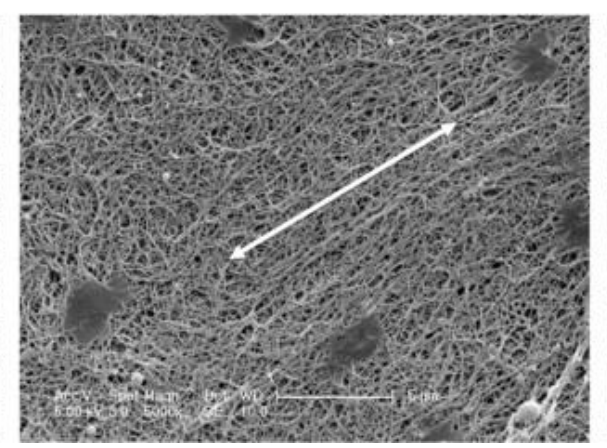

EDGE

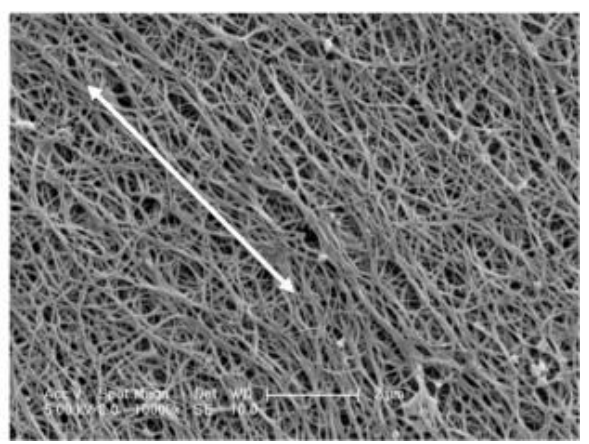

